\title{
A Practical Approach to Detect Faults of Marine Diesel Engine
}

\author{
Zhengyang Qi, Yunsong Qi, Guangpeng Hu \\ School of Computer, Jiangsu University of Science and Technology, Zhenjiang, China \\ Email:296913335@qq.com, mailqys@163.com
}

How to cite this paper: Qi, Z.Y., Qi, Y.S and Hu, G.P. (2020) A Practical Approach to Detect Faults of Marine Diesel Engine. Journal of Computer and Communications, 8, 12-21.

https://doi.org/10.4236/jcc.2020.88002

Received: July 17, 2020

Accepted: August 4, 2020

Published: August 7, 2020

Copyright $\odot 2020$ by author(s) and Scientific Research Publishing Inc. This work is licensed under the Creative Commons Attribution International License (CC BY 4.0).

http://creativecommons.org/licenses/by/4.0/

(c) (i) Open Access

\begin{abstract}
The existing marine diesel engine fault diagnosis methods mainly have the problems of model complexity, large amount of calculation, and unable to carry out real-time fault diagnosis of diesel engine. In this paper, a simple and practical approach to detect faults of marine diesel engine is studied. According to a set of sensing data, the fitting equation of each parameter changing with the running state of diesel engine was fitted statistically. Then, the threshold range of each parameter changing with the running state of diesel engine was fitted. During fault diagnosis, the real-time parameters of the sensor in the current running state were calculated according to the real-time running data. If the parameters exceed the threshold range, it is abnormal operation. Because the sensor signal corresponds to the operation status of each specific component, the abnormal evaluation directly indicates the specific fault. Experimental results show that the method has a good practical effect.
\end{abstract}

\section{Keywords}

Condition-Based Maintenance, Fault Detection, Marine Diesel Engine

\section{Introduction}

With the increasingly high performance and complex structure of modern ship system, the diesel generator set of ship power station is also developing towards the direction of large-scale, high-speed and precision, with continuous improvement of working performance and higher degree of automation. On the one hand, it will greatly improve the labor productivity, improve the power quality of the ship system, and reduce the maintenance cost and energy consumption. But on the other hand, once a part or a link breaks down, the whole ship system will be paralyzed, which will directly or indirectly cause huge economic losses, even cause damage to key equipment and endanger personal safety. Therefore, it 
is of great significance to judge the cause of the fault quickly and to eliminate the fault effectively to ensure the normal navigation of the ship.

In the existing marine diesel engine fault diagnosis system, fault tree analysis, thermodynamic model, vibration signal processing, neural network model and other diagnostic modes are more representative: fault tree analysis method [1] [2] through observation and the use of measurement tools to check the diesel engine fault, combined with expert experience and knowledge to achieve the purpose of troubleshooting. Although the method is simple, it requires a high level of knowledge accumulation for inspectors, so it has great limitations. It is usually used in combination with other diagnostic methods. The thermodynamic model method [3] [4] [5] collects and analyzes the thermodynamic parameters of marine diesel engine, and judges the operation state of diesel engine according to its changes. At present, the research of this method is mainly through the simulation of the working process of the diesel engine to study the influence of different faults on the performance parameters of the diesel engine. The main disadvantage is that the scientificity of the model is especially important to the effect of fault diagnosis, and the determination of model parameters is also a topic worthy of study. The vibration signal processing method [6] [7] is to extract and analyze the vibration signals during the operation of marine diesel engine to determine the operation status of diesel engine. The main vibration information of marine diesel engine includes the vibration of fixed parts, torque vibration of moving parts and pressure fluctuation. However, the vibration analysis method usually cannot provide the actual cause of the fault, and the measurement and processing of the vibration signal is complex. Neural network model [8] fault diagnosis technology is a new generation of artificial intelligence method. It can automatically obtain the system model by learning various operation parameters of marine diesel engine. Although there are many research papers in this field, however, the theory and learning algorithm of neural network still need to be further improved and improved. Moreover, this method has many problems, such as low diagnosis efficiency, low transparency of diagnosis results, and difficult to determine the specific fault location.

To sum up, the existing marine diesel engine fault diagnosis methods mainly have the problems of model complexity, large amount of calculation, and unable to carry out real-time fault diagnosis of diesel engine.

In this paper, a simple and practical method of diesel engine fault diagnosis is studied. According to a set of sensing data, the fitting equation of each parameter changing with the running state of diesel engine is fitted statistically. Then, the threshold range of each parameter changing with the running state of diesel engine is fitted. During fault diagnosis, the real-time parameters of the sensor in the current running state are calculated according to the real-time running data. If the parameters exceed the threshold range, it is abnormal operation. Because the sensor signal corresponds to the operation status of each specific component, the abnormal evaluation directly indicates the specific fault. The proposed method is simple and practical and suitable for real-time fault diagnosis. Experi- 
mental results show that the method has a good practical effect.

\section{Method Description}

\subsection{Problem Description}

Marine diesel engine can be divided into six main subsystems: combustion system, cooling system, lubrication system, fuel injection system, gas system, electrical system, etc. To monitor the engine, each system is equipped with a specific list of sensors. For example, temperature, pressure, flow, speed, torque and voltage sensors. When the diesel engine is working, the gas temperature is as high as $1800^{\circ} \mathrm{C}$ [9], which makes the cylinder head, cylinder sleeve, piston, air valve, fuel injector and other parts in direct contact with the gas heated seriously. To explain the problem conveniently, this paper takes a group of sensor parameters related to exhaust temperature of marine diesel engine as an example to illustrate the use method of this paper. It should be noted that to describe the principle of the fault diagnosis method in this section more clearly, this paper simplifies the selection of a group of relevant data. These data include engine load power, engine speed, scavenge volume, scavenge pressure, exhaust temperature, etc.

\subsection{Sensor State Equation}

The data of each sensor is correlated. The relationship between the theoretical estimate value $\hat{y}$ of a certain sensor and the observed value $x_{i}(i=1,2, \cdots, r)$ of other $r$ sensors is analyzed as follows.

$$
\hat{y}=\hat{\theta}_{0}+\sum_{i=1}^{r}\left(\hat{\theta}_{1, i} x_{i}+\hat{\theta}_{2, i} x_{i}^{2}\right)
$$

Parameter $\hat{\theta}_{0}$ is the offset, and $\hat{\theta}_{1, i}, \hat{\theta}_{2, i}$ is the coefficients of the 1st and 2nd components of the $i$ th sensor variables. To facilitate regression calculation, linearize formula (1) and replace all higher-order variables with a new primary variable, then the theoretical estimation $\hat{y}$ of sensor y can be expressed as the following relationship:

$$
\hat{y}=\hat{\alpha}_{0}+\sum_{i=1}^{n} \hat{\alpha}_{i} z_{i}
$$

An independent variable $x_{i}$ and its square $x_{i}^{2}$ in formula (1) are represented by two independent variables in formula (2), so the value of $n$ is $2 * r$. For example, $x_{1}$ corresponds to $z_{1}, x_{1}^{2}$ corresponds to $z_{2}, x_{2}$ corresponds to $z_{3}$, $x_{2}^{2}$ corresponds to $x_{4}, \ldots$, and so on. Different from formula (1), the regression coefficient is represented by $\alpha_{i}(i=0,1,2, \cdots, n)$, and there are $n+1$ parameters, in which $\hat{\alpha}_{0}$ is the offset.

The reason why formula (1) and formula (2) choose the quadratic regression model is that the diagnosed object is a large time-delay object, and the quadratic curve is enough to show its change rate. Of course, the above model needs to be corrected after calculation.

To correct the model, the key term $T$-test is carried out for the model shown 
in formula (1). If the $p$-value of an item is greater than 0.05 , it is considered that the item is not significant in the model, and it can be removed from the regression model. Then recalculate the regression model, and finally carry out the $F$-test on the model. The smaller the significance of $F$-test, the better the model effect. In general, the significance of $F$-test should be less than 0.05 .

If the key term $T$-test and the whole model $F$-test are passed, the regression ability of the model is relatively good.

\subsection{Regression Parameter Estimation}

The regression parameters of formula (2) can be estimated by the minimum variance criterion. The observation value of sensor $y$ and the observation value of sensor $x$ (make linear transformation according to the corresponding relationship between formula (1) and (2) are represented by $m$ samples as follows:

$$
\left(x_{k, 1}, x_{k, 2}, \cdots, x_{k, n}, y_{k}\right)(k=1,2, \cdots, m)
$$

According to the principle of least square, we have the following loss function:

$$
Q=\sum_{i=1}^{m}\left(y_{i}-\left(\hat{\alpha}_{0}+\sum_{j=1}^{n} \hat{\alpha}_{j} x_{i}\right)\right)^{2}
$$

To minimize the loss function, The regression coefficient $\mathrm{X}$ shall satisfy the following equations:

$$
\left\{\begin{array}{c}
\frac{\partial}{\partial \hat{\alpha}_{0}} Q=0 \\
\frac{\partial}{\partial \hat{\alpha}_{1}} Q=0 \\
\frac{\partial}{\partial \hat{\alpha}_{2}} Q=0 \\
\vdots \\
\frac{\partial}{\partial \hat{\alpha}_{n}} Q=0
\end{array}\right.
$$

Than we can get the normal equations:

$$
\left\{\begin{array}{c}
\sum_{k=1}^{m}\left(\hat{\alpha}_{0}+\hat{\alpha}_{1} x_{1 k}+\hat{\alpha}_{2} x_{2 k}+\cdots+\hat{\alpha}_{n} x_{n k}\right)=\sum_{k}^{m} y_{k} \\
\sum_{k=1}^{m}\left(\left(\hat{\alpha}_{0}+\hat{\alpha}_{1} x_{1 k}+\hat{\alpha}_{2} x_{2 k}+\cdots+\hat{\alpha}_{n} x_{n k}\right) x_{1 k}\right)=\sum_{k}^{m}\left(y_{k} x_{1 k}\right) \\
\sum_{k=1}^{m}\left(\left(\hat{\alpha}_{0}+\hat{\alpha}_{1} x_{1 k}+\hat{\alpha}_{2} x_{2 k}+\cdots+\hat{\alpha}_{n} x_{n k}\right) x_{2 k}\right)=\sum_{k}^{m}\left(y_{k} x_{2 k}\right) \\
\vdots \\
\sum_{k=1}^{m}\left(\left(\hat{\alpha}_{0}+\hat{\alpha}_{1} x_{1 k}+\hat{\alpha}_{2} x_{2 k}+\cdots+\hat{\alpha}_{n} x_{n k}\right) x_{n k}\right)=\sum_{k}^{m}\left(y_{k} x_{n k}\right)
\end{array}\right.
$$

Solving equations in (4), we can get the estimated parameter values $\hat{\alpha}_{i}, i=0,1,2, \cdots, n$.

\subsection{Real Time Fault Diagnosis}

In real-time fault diagnosis, the data of each sensor is estimated according to formula (2). If the deviation between the observed value and the estimated value 
of a sensor exceeds a certain threshold, the observed value of the sensor may indicate a fault. The specific fault conditions corresponding to the observed values of sensors need to be cross verified according to the observed values of other relevant sensors. For example, if the exhaust temperature is too high and the scavenging pressure is too low, it may be that the supercharger suction filter is blocked.

\section{Experiment and Result Analysis}

\subsection{Data Set}

In the project, MAN B\&W $6 \mathrm{~S} 42$ diesel engine is used to test the operation data of the real ship. The number of original data samples is 15,800 , and Table 1 is a representative sample of the preprocessed data. The meaning of each column of data in Table 1 is as follows:

$A$ : Exhaust temperature $\left({ }^{\circ} \mathrm{C}\right)$,

$B$ : Power (kw),

$C$ : Sweeping gas volume $(\mathrm{kkg} / \mathrm{h})$,

$D$. Scavenging pressure (bar abs),

E: Speed $(\mathrm{kr} / \mathrm{min})$

For the convenience of data processing, the unit of sweep gas volume and rotation speed is divided by 1000 according to the original data, and the unit is also transformed accordingly.

\subsection{Model Fitting}

Figure 1 shows the trend of the data in Table 1. As can be seen from Figure 1,

Table 1. Sample of experimental data.

\begin{tabular}{cccccc}
\hline Number & $A$ & $B$ & $C$ & $D$ & $E$ \\
\hline 1 & 238.5 & 6.48 & 54.95 & 3.99 & 136 \\
2 & 231.7 & 6.156 & 53.05 & 3.81 & 133.7 \\
3 & 226.3 & 5.832 & 51.2 & 3.64 & 131.3 \\
4 & 222.3 & 5.508 & 49.2 & 3.46 & 128.8 \\
5 & 219.7 & 5.184 & 47.15 & 3.29 & 126.3 \\
6 & 218.5 & 4.86 & 44.95 & 3.12 & 123.6 \\
7 & 218.7 & 4.536 & 42.7 & 2.94 & 120.8 \\
8 & 220.3 & 4.212 & 40.3 & 2.77 & 117.8 \\
9 & 223.3 & 3.888 & 37.75 & 2.59 & 114.7 \\
10 & 227.7 & 3.564 & 35.1 & 2.42 & 111.4 \\
11 & 233.5 & 3.24 & 32.3 & 2.24 & 107.9 \\
12 & 241.6 & 2.916 & 29.4 & 2.07 & 104.2 \\
13 & 251.1 & 2.592 & 26.45 & 1.9 & 100.2 \\
14 & 259.6 & 2.268 & 23.3 & 1.73 & 95.8 \\
\hline
\end{tabular}




\section{Trend chart of sensor observations}

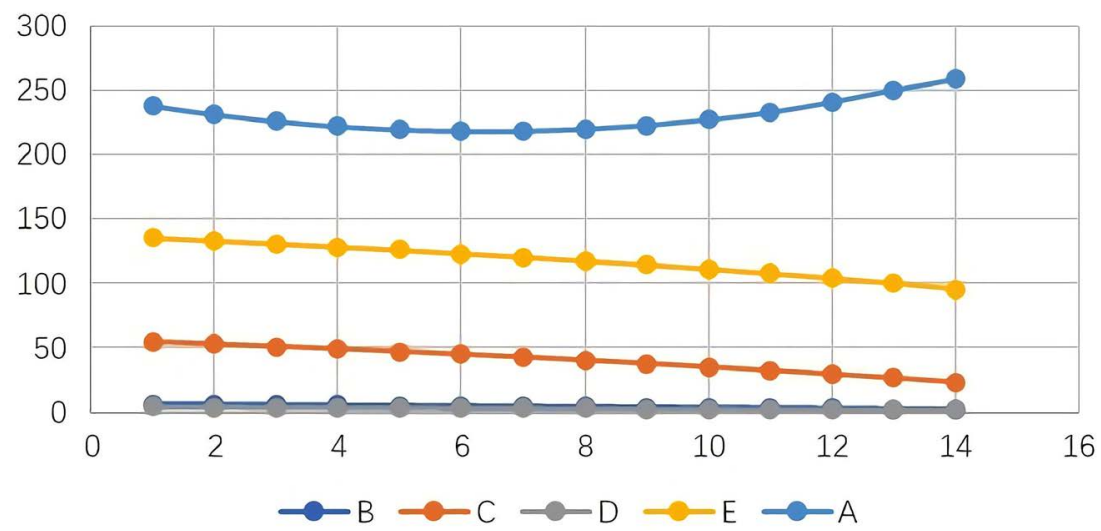

Figure 1. Trend chart of data change.

the trend of data change in Table 1 is relatively gentle, and the quadratic curve is enough to fit these experimental data. As an example, the non-linear relationship between exhaust temperature and other index data is fitted.

According to Table 1, the nonlinear relationship between exhaust temperature and other index data is set as follows:

$$
A=\alpha_{0}+\alpha_{1} B+\alpha_{2} C+\alpha_{3} D+\alpha_{4} E+\alpha_{5} B^{2}+\alpha_{6} C^{2}+\alpha_{7} D^{2}+\alpha_{8} E^{2}
$$

Equation (7) is fitted with the least square method. See Table 2 and Table 3 for model fitting results and residual analysis. The model correlation index $R^{2}=0.999835, F$-test significance is $5.01 \times 10^{-9}$, indicating that the fitting effect is good.

\subsection{Fault Diagnosis Case}

In this study, a group of observed data of sensors is $\{A=231.42, B=4.86, C=$ $44.95, D=2.92, E=123.6\}$.

According to the parameters in formula (7) and Table 2, the estimated exhaust temperature $A$ is:

$$
\begin{aligned}
A= & -1310.889-356.5483 B-16.5431 C+416.0629 D+30.3444 E \\
& +28.30363134 B^{2}+0.2624 C^{2}-68.8581 D^{2}-0.1029 E^{2} \\
= & 218.2922
\end{aligned}
$$

Similarly, the estimation models and values of other sensors are as follows:

$$
\begin{aligned}
B= & 4.8092+0.0269 A+0.0976 C-0.3323 D-0.1633 E \\
& -0.0004 C^{2}+0.0656 D^{2}+0.0001 E^{2} \\
= & 4.8536 \\
C= & -22.0582-0.2698 A+14.0324 B-4.1778 D+1.0636 E \\
+ & 0.0006 A^{2}-0.5644 B^{2}+0.9562 D^{2}-0.0056 E^{2} \\
= & 44.6262 \\
D= & 1.2329-0.002 A+0.0078 B+0.0307 C-0.0124 E \\
& +0.0138 B^{2}+0.0001 E^{2} \\
= & 3.1197
\end{aligned}
$$


Table 2. Model fitting results.

\begin{tabular}{cccc}
\hline & Parameter value & t-statistics & P-value \\
\hline$\alpha_{0}$ & -1310.89 & -3.13071 & 0.025937 \\
$\alpha_{1}$ & -356.548 & -3.61718 & 0.015265 \\
$\alpha_{2}$ & -16.5431 & -4.14201 & 0.008979 \\
$\alpha_{3}$ & 416.0629 & 2.360033 & 0.064748 \\
$\alpha_{4}$ & 30.34442 & 3.806339 & 0.012547 \\
$\alpha_{5}$ & 28.30363 & 3.166967 & 0.0249 \\
$\alpha_{6}$ & 0.262391 & 4.19722 & 0.008512 \\
$\alpha_{7}$ & -68.8581 & -2.37225 & 0.063775 \\
$\alpha_{8}$ & -0.10286 & -2.5849 & 0.049139 \\
\hline
\end{tabular}

Table 3. Residual analysis table.

\begin{tabular}{ccc}
\hline Observed value & Forecast $y$ & Residual \\
\hline 238.5 & 238.5657 & -0.06574 \\
231.7 & 231.5996 & 0.100376 \\
226.3 & 226.2581 & 0.041882 \\
222.3 & 222.3203 & -0.02034 \\
219.7 & 219.9023 & -0.2023 \\
218.5 & 218.3242 & 0.175755 \\
218.7 & 218.8018 & -0.10176 \\
220.3 & 220.2416 & 0.058361 \\
223.3 & 223.0585 & 0.241507 \\
227.7 & 227.9544 & -0.25439 \\
233.5 & 233.4965 & 0.003489 \\
241.6 & 241.7613 & -0.16135 \\
251.1 & 250.8081 & 0.291866 \\
259.6 & 259.7074 & -0.10736 \\
\hline
\end{tabular}

$$
\begin{aligned}
E= & 57.3831+30.4189 B+0.735 C-29.0157 D-0.0256 A \\
& -2.0215 B^{2}-0.0137 C^{2}+4.9585 D^{2}+0.0002 A^{2} \\
= & 124.1394
\end{aligned}
$$

Note that the coefficient of $A^{2}$ term in the above estimation model of sensor $B$ is very small and ignored; the coefficient of $A^{2}, C^{2}$ term in the estimation model of sensor $D$ is very small and ignored;

The estimated value of each sensor represents the normal level of the sensor, and the deviation of $5 \%$ between the observed value and the estimated value is abnormal. Table 4 shows the test results of the test sample data.

The test results in Table 4 show that the exhaust gas temperature a is too high, the scavenging pressure $d$ is too low. The main reasons for the high exhaust gas 
Table 4. Test results of sample data.

\begin{tabular}{cccccc}
\hline & $A$ & $B$ & $C$ & $D$ & $E$ \\
\hline Observed value & 231.42 & 4.85 & 44.97 & 2.92 & 124.2 \\
Estimate & 218.2922 & 4.8536 & 44.6262 & 3.1197 & 124.1394 \\
Estimate 1.05 & 229.2068 & 5.09628 & 46.85751 & 3.275685 & 130.3464 \\
Estimate 0.95 & 207.3776 & 4.61092 & 42.39489 & 2.963715 & 117.9324 \\
Abnormal or not & Too high & Normal & Normal & Too low & Normal \\
\hline
\end{tabular}

temperature are late fuel supply time, poor atomization of fuel injector, blockage of air filter, serious overload, etc. The reasons for the low scavenging pressure are air filter blockage, turbocharger failure, air leakage in intake pipe, unequal intake phase. With other indicators are normal, according to experience, if the exhaust temperature is too high and the scavenging pressure is too low, it is possible that the supercharger suction filter is blocked.

\section{Conclusions}

In this paper, the modeling method of signal parameter estimation for marine diesel engine under complex conditions is studied. Using these estimated values, we can judge whether the observed values of each signal parameter are abnormal, and then cross diagnose the system fault. Experimental results show that the method is feasible and robust.

The research in this paper is only for method discussion, not for diesel engine working principle discussion. For example, from the perspective of the whole system, the exhaust temperature is too higher and the hot gas pressure is too low. The reason is not necessarily the filter fouling at the suction port of the supercharger, but also the pipeline fouling, or even the low ambient air pressure, the abnormal oxygen content of the ambient air, etc. This paper focuses on the feasibility of the method. Theoretically, in the case of sufficient sensing parameters, the problem of fault diversity under the same abnormal signal symptoms can be effectively solved by extending the method of this paper to the joint analysis of the whole system data. Although the marine diesel engine system is complex, each subsystem has certain independence, and each signal parameter also has redundancy. A parameter signal estimation model does not need too many parametric signals. Therefore, the estimation of each signal parameter can be independently modeled in a small range, and the fault diagnosis and confirmation can be comprehensively evaluated by the evaluation results of each sub model. The relationship between the parameter signals and the fault is generally determined by the machine learning algorithm such as the expert or the decision tree based on the sample data.

This paper presents a practical method for real-time fault diagnosis of diesel engine. The experiment is only verified in the local subsystem. There are two abnormal data in the sample data observation in Table 4 of this paper, the esti- 
mation model is based on the comprehensive evaluation of various data, and the deviation of a small number of observation values does not bring too much impact on the estimation of normal indicators. In fact, diesel engine is a complex system including fuel system, crank-piston system, combustion chamber, turbocharging system, starting-reversing system, cooling system, lubrication system [10]. If the number of outliers in the sample data increases further, what impact will these outliers have on the estimation of system model and how to correct them will be our further research content.

\section{Conflicts of Interest}

The authors declare no conflicts of interest regarding the publication of this paper.

\section{References}

[1] Ünver, B., Gürgen, S., Sahin, B., et al. (2019) Crankcase Explosion for Two-Stroke Marine Diesel Engine by Using Fault Tree Analysis Method in Fuzzy Environment. Engineering Failure Analysis, 97, 288-299. https://doi.org/10.1016/j.engfailanal.2019.01.007

[2] Liu, H., Shah, S. and Jiang, W. (2004) On-Line Outlier Detection and Data Cleaning. Computers \& Chemical Engineering, 28, 1635-1647. https://doi.org/10.1016/j.compchemeng.2004.01.009

[3] Lazakis, I., Gkerekos, C. and Theotokatos, G. (2019) Investigating an SVM-Driven, One-Class Approach to Estimating Ship Systems Condition. Ships and Offshore Structures, 14, 432-441. https://doi.org/10.1080/17445302.2018.1500189

[4] Rubio, J.A.P., Vera, G.F., Grau, J.H., et al. (2018) Marine Diesel Engine Failure Simulator Based on Thermodynamic Model. Applied Thermal Engineering, 144, 982995. https://doi.org/10.1016/j.applthermaleng.2018.08.096

[5] Zhu, Y., Xia, C., Shreka, M., et al. (2020) Combustion and Emission Characteristics for a Marine Low-Speed Diesel Engine with High-Pressure SCR System. Environmental Science and Pollution Research, 27, 12851-12865. https://doi.org/10.1007/s11356-019-04194-2

[6] Li, Z.X., Jiang, Y., Duan, Z.H., et al. (2018) A New Swarm Intelligence Optimized Multiclass Multi-Kernel Relevant Vector Machine: An Experimental Analysis in Failure Diagnostics of Diesel Engines. Structural Health Monitoring, 17, 1503-1519. https://doi.org/10.1177/1475921717746735

[7] Nag, S., Sharma, P., Gupta, A., et al. (2019) Combustion, Vibration and Noise Analysis of Hydrogen-Diesel Dual Fuelled Engine. Fuel, 241, 488-494.

https://doi.org/10.1016/j.fuel.2018.12.055

[8] Yun, Q.S., Zhang, C. and Ma, T. (2019) Fault Diagnosis of Diesel Generator Set Based on Deep Believe Network. Proceedings of the 2 nd International Conference on Artificial Intelligence and Pattern Recognition, August 2019, 186-190. https://doi.org/10.1145/3357254.3358601

[9] Xu, H., Liu, S., Yin, B., et al. (2018) Visualization of Combustion Performance and Emission Characteristics of a Four-Cylinder Diesel Engine at Various Inlet Oxygen Concentrations at Part Loads. Journal of Mechanical Science and Technology, 32, 2925-2934. https://doi.org/10.1007/s12206-018-0549-2

[10] Charchalis, A. and Pawletko, R. (2011) Application of Artificial Intelligence Me- 
thods for the Diagnosis of Marine Diesel Engines. International Conference on Computational Collective Intelligence, Springer, Berlin, Heidelberg, 261-270.

https://doi.org/10.1007/978-3-642-23938-0_27 